\title{
COVID-19 Image Segmentation Based on Deep Learning and Ensemble Learning
}

\author{
Philip MEYER ${ }^{\mathrm{a}}$, Dominik MÜLLER ${ }^{\mathrm{a}, 1}$, Iñaki SOTO-REYa and Frank KRAMER ${ }^{\mathrm{a}}$ \\ a IT-Infrastructure for Translational Medical Research, University of Augsburg
}

\begin{abstract}
Medical imaging offers great potential for COVID-19 diagnosis and monitoring. Our work introduces an automated pipeline to segment areas of COVID-19 infection in CT scans using deep convolutional neural networks. Furthermore, we evaluate the performance impact of ensemble learning techniques (Bagging and Augmenting). Our models showed highly accurate segmentation results, in which Bagging achieved the highest dice similarity coefficient.
\end{abstract}

Keywords. COVID-19, segmentation, ensemble learning, computed tomography, deep learning, artificial intelligence

\section{Introduction}

Multiple studies have shown that computed tomography imaging of the thorax is more sensitive for COVID-19 screening compared to RT-PCR testing [1]. Thus, our aim was to build an automated pipeline in order to segment COVID-19 related abnormalities on CT scans using deep convolutional neural networks and ensemble learning.

\section{Methods}

The pipeline was based on our in-house developed framework MIScnn [2]. We utilized the COVID-19 Lung CT Lesion Segmentation Challenge 2020 dataset containing 245 CT scans into a pre-defined training and testing dataset consisting of 199 and 46 samples, respectively ${ }^{2}$. The following preprocessing methods were applied: Clipping of Hounsfield units to [-1250, 255], normalization to unsigned byte range and, finally, resampling the $\mathrm{CT}$ scans to a uniform voxel spacing of $1.58 \times 1.58 \times 2.70 \mathrm{~mm}$. We utilized the standard U-Net architecture for training and prediction on overlapping chunks of each image as described in $[3,4]$.

We established the created pipeline as baseline and experimented with augmenting and bagging as ensemble learning techniques. Augmenting describes the process of data augmentation on the inference dataset. We applied horizontal mirroring as inference augmentation method which resulted in 4 predictions per sample. We combined these by majority rule, with ties resulting in a positive identification. Bagging is defined as generating multiple predictions using a collective of models of

\footnotetext{
${ }^{1}$ Corresponding Author: Dominik Müller, IT Infrastructure for Translational Medical Research, Alter Postweg 101, 86159 Augsburg, Germany; E-mail: dominik.mueller@informatik.uni-augsburg.de.

${ }^{2}$ https://covid-segmentation.grand-challenge.org/COVID-19-20/
} 
the same type. The model collective was created by performing a 5-fold crossvalidation on the training dataset ( $80 \%$ training and $20 \%$ validation subsets). The resulting 5 predictions for each scan were combined by using majority rule, as well.

\section{Results}

We computed Dice similarity coefficients (DSC) on the testing set resulting in 0.63 , $0.64,0.68$ for baseline, augmenting and bagging, respectively. It can be seen in figure 1 , augmenting showed a small improvement on the average DSC. However, the bagging pipeline revealed more robust predictions while having increased performance.

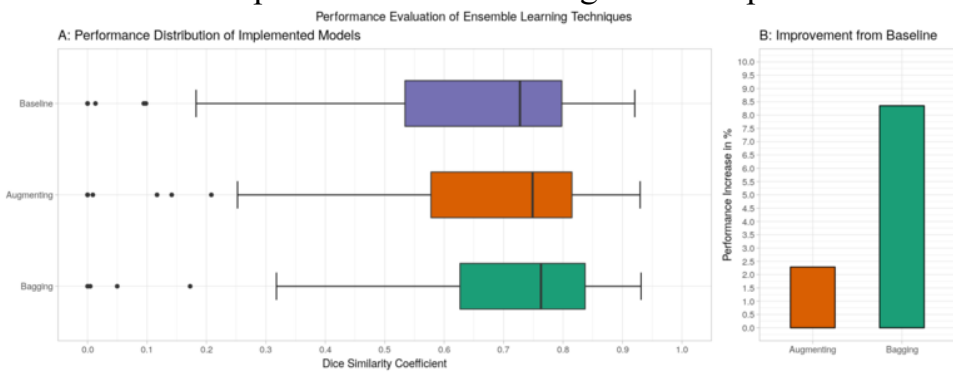

Figure 1. (A) Performance distribution measured by the DSC on a separated testing set.

(B) Average performance increase compared to the baseline performance.

\section{Discussion}

We showed that our pipeline is able to produce robust prediction results for segmentations of COVID-19 infected regions. In the future, we plan to further analyze ensemble learning impact on segmentation.

\section{Conclusions}

To summarize, we demonstrated that deep learning based medical image segmentation can be a powerful assistant to quantitative disease detection and monitoring. We are working on extending this analysis to consider more variation in the application of ensemble learning methods.

\section{References}

[1] Ai T, et.al. Correlation of chest CT and RT-PCR testing for coronavirus disease 2019 (COVID-19) in China: a report of 1014 cases. Radiology. 2020 Aug;296(2):E32-40.

[2] Müller D, et.al. MIScnn: a framework for medical image segmentation with convolutional neural networks and deep learning. BMC Medical Imaging. 2021 Dec;21(1):1-1.

[3] Ronneberger O, et.al. U-net: Convolutional networks for biomedical image segmentation. InInternational Conference on Medical image computing and computer-assisted intervention 2015 Oct 5 (pp. 234-241). Springer, Cham.

[4] Minaee S, et.al. Image segmentation using deep learning: A survey. IEEE Transactions on Pattern Analysis and Machine Intelligence. 2021 Feb 17. 